SUBJECT AREAS:

MECHANICAL

PROPERTIES

GLASSES

Received

15 October 2013

Accepted

9 January 2014

Published

28 January 2014

Correspondence and requests for materials should be addressed to G.W. Ig.wang@shu. edu.cn) \title{
evolution and strength in a bulk metallic glass at cryogenic temperature
}

\author{
J. Tan', G. Wang' , Z. Y. Liu', J. Bednarčík', Y. L. Gao', Q. J. Zhai' , N. Mattern³ \& J. Eckert3,4
}

\begin{abstract}
'Laboratory for Microstructures, Shanghai University, 200444 Shanghai, China, ${ }^{2}$ HASYLAB at DESY, Notkestr. 85, D-22603 Hamburg, Germany, ${ }^{3}$ IFW Dresden, Institute for Complex Materials, D-01069 Dresden, Germany, ${ }^{4}$ TU Dresden, Institute of Materials Science, D-01062 Dresden, Germany.
\end{abstract}

A model $\mathrm{Zr}_{41.25} \mathrm{Ti}_{13.75} \mathrm{Ni}_{10} \mathrm{Cu}_{12.5} \mathrm{Be}_{22.5}$ (at.\%) bulk metallic glass (BMG) is selected to explore the structural evolution on the atomic scale with decreasing temperature down to cryogenic level using high energy $\mathrm{X}$-ray synchrotron radiation. We discover a close correlation between the atomic structure evolution and the strength of the BMG and find out that the activation energy increment of the concordantly atomic shifting at lower temperature is the main factor influencing the strength. Our results might provide a fundamental understanding of the atomic-scale structure evolution and may bridge the gap between the atomic-scale physics and the macro-scale fracture strength for BMGs.

ulk metallic glasses (BMGs) exhibit high strength and hardness ${ }^{1-3}$, which is believed to be associated with the cohesive strength between atoms and/or atomic clusters ${ }^{4-6}$. In the past few decades, a lot of attempts have been devoted to reveal the primary deformation units and to elucidate yielding or fracture behavior as well as the plastic deformation mechanisms in BMGs by theoretic analysis ${ }^{5,7,8}$, experiments ${ }^{9,10}$, and simulation ${ }^{11}$. Since it is difficult to directly observe these so-called deformation units, some work investigated the dynamical response of atomic clusters through modifying the loading modes and the environmental temperatures ${ }^{9,10,12}$, attempting to explore how the deformation units affect the plastic deformation. More recently, it was found that when the temperature was decreased to cryogenic level, a freezing effect could render deformation units, such as shear transition zones (STZ), changed in size and activation energy ${ }^{13}$. Moreover, cryogenic temperature also improves the yield strength and plasticity of $\mathrm{BMGs}^{14-17}$. Despite intensive research work on these atomic clusters (STZs or free volume arrangements), a direct observation of the structural evolution at the cryogenic temperature has not been done so far. An accurate physical mechanism quantitatively describing the relationship between the deformation units and the strength of BMGs at cryogenic temperature is still unclear.

In this work, a $\mathrm{Zr}_{41.25} \mathrm{Ti}_{13.75} \mathrm{Ni}_{10} \mathrm{Cu}_{12.5} \mathrm{Be}_{22.5}$ (at.\%) BMG was selected as a model material to explore the structural evolution on the atomic scale with decreasing temperature down to cryogenic level. In-situ observation at cryogenic temperature was conducted by synchrotron high energy X-ray diffraction ${ }^{18,19}$. The atomic structure in terms of short- and medium-range order was analyzed. A concept of a concordant region was used to elucidate the correlation between the atomic behavior and the strength of the BMG.

\section{Results}

Diffraction intensity curves, $I(q)$, at eight different temperatures show the typical diffuse scattering rings representing the glassy nature of the specimens at low temperatures (Fig. 1a). Enlarged the first maximum of $I(q)$, shown in the inset of Fig. 1a, suggests that the first maximum position shifts to higher $q$ values with decreasing temperature. After polarization processing, sample absorption and inelastic scattering correcting intensity curves $^{20}$, the total structure factors, $S(q)$, at eight temperatures, are calculated to be shown in Fig. 1b. Enlarged first maximum of $S(q)$, shown in the inset of Fig. 1b, indicates obvious differences at the temperatures ranged from $98 \mathrm{~K}$ to $308 \mathrm{~K}$. The first diffuse diffraction peak carries significant information about the average atomic volume and extension of atomic correlations or degree of order in $\mathrm{BMGs}^{20}$, which can reflect the structure information in the medium-range order (Fig. S1) ${ }^{21}$.

To further quantitatively describe the change in the first maximum of $S(q)$, the position and the full width at half maximum (FWHM) of the first maximum at different temperatures are calculated by Gaussian function fitting, taking $98 \mathrm{~K}$ as an example shown in Fig. 1c, which reveals an increasing trend for the position from 2.6643 

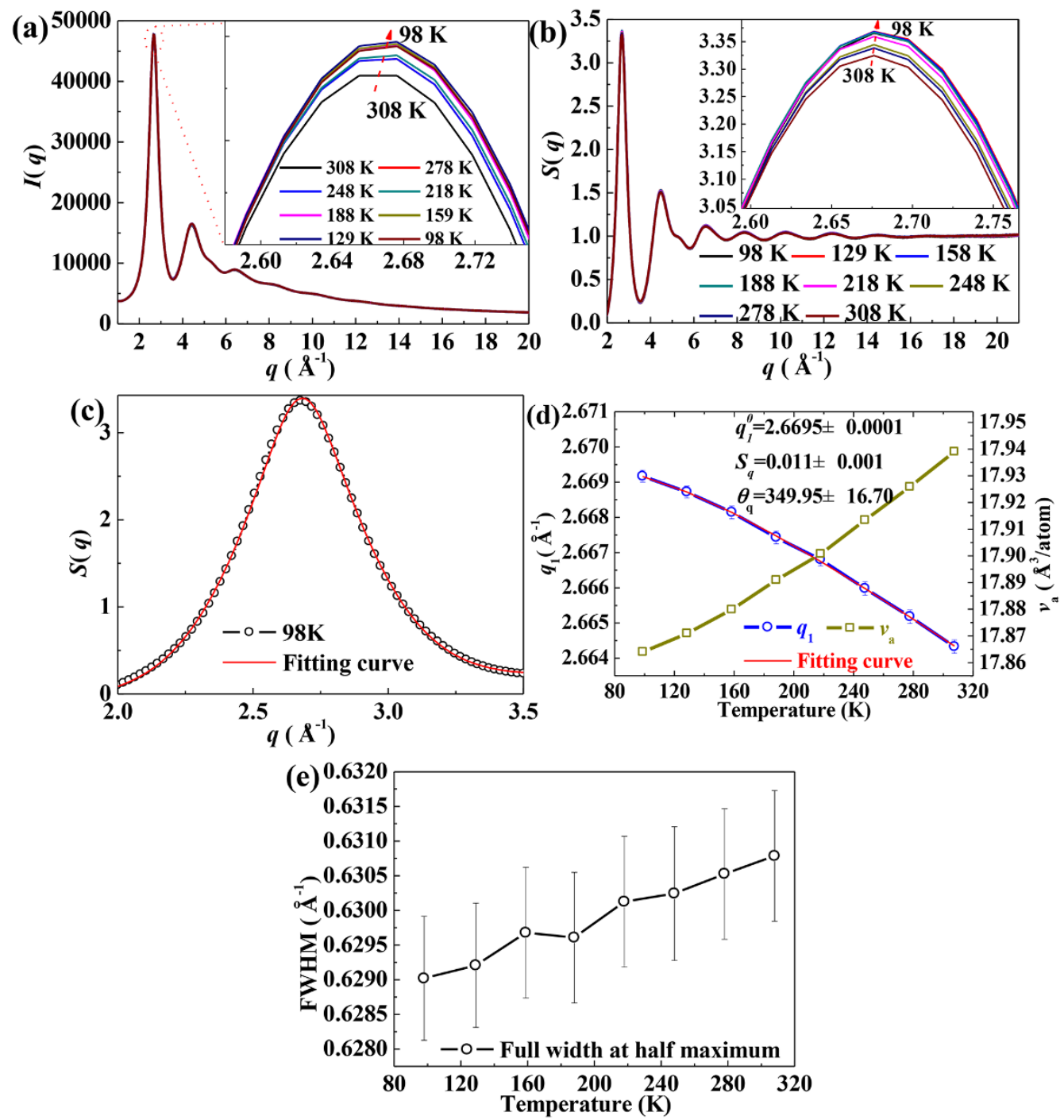

Figure $1 \mid$ Structural evolution in reciprocal space. (a) Diffraction intensity, $I(q)$, at different temperatures. Inset shows the enlarged first maximum. (b) Structure factors at different temperatures. Inset shows the enlarged first maximum. (c) Gaussian fitting curve for the first maximum at 98 K. (d) First maximum position and averaged atomic volume of the BMG as a function of temperature, indicating shrinkage with decreasing temperature.

Red line shows that the Varshni equation can well fit the first maximum position in the temperature range of $98 \mathrm{~K}$ to $380 \mathrm{~K}$. (e) Full width at half maximu of the first maximum of diffraction intensity curve as a function of temperature.

$\pm 0.0002 \AA^{-1}$ to $2.6692 \pm 0.0002 \AA^{-1}$ with decreasing temperature from $308 \mathrm{~K}$ to $98 \mathrm{~K}$ (Fig. 1d). It is obvious that the profile fit results are used in the current study because the maximum positions can be determined by whole pattern fitting with high accuracy (standard deviation of $\pm 0.0002 \AA^{-1}$ is estimated for the first maximum position at $q_{1}$ (Fig. 1c)). The absolute error (estimated to be approximately $0.002 \AA^{-1}$ ) of the positions is much larger. However, only the relative maximum positions are of important for the analysis of shrinkage behavior. Figure 1e plots the FWHM of the first maximum as a function of temperature, which shows that the first maximum is sharpened due to the decrease in temperature. Since the error range is larger than the change of the FWHM (Fig. 1e), the accurate value of the FWHM is not possible. However, we can deduce that the first maximum has a trend of sharpening, corresponding to the degree of disorder in the glassy phase decreases due to cooling shrinkage.

The first maximum position of structure factor, $q_{1}$, versus atomic volume, $v_{\mathrm{a}}$, has been demonstrated to follow a power-law scaling relationship, i.e., $q_{1} \cdot v_{\mathrm{a}}^{0.433}=9.3$ because the medium-range order of BMGs at room temperature has the characteristics of a fractal network $^{22}$. In the present study, although the BMG is cooled down to the cryogenic level, the structure of the BMG is still in a fully glassy state (Fig. 1a). Thus, it is rational to use the power-law scaling relation of $q_{1} \cdot v_{\mathrm{a}}^{0.433}=9.3$ to estimate the average atomic volume of the BMG at lower temperatures, which is plotted in Fig. 1d. According to the expectations of macroscopic thermal expansion, it is evident that the atomic volume of the BMG decreases from approximately 17.94 $\AA^{3}$ /atom to $17.86 \AA^{3}$ /atom with decreasing temperature.

Figure 1d clearly shows that the position of the first maximum of $S(q)$ and the atomic volume do not behave a linear manner with decreasing temperature. In solids, the atomic volume depends on the frequency of lattice vibration. The coefficient of thermal expansion (or cooling shrinkage) is proportionate to the lattice specific heat $^{23}$. Thus, the enthalpy of solid, which is an integral of the lattice specific heat, is proportional to the temperature dependent volume change. The lattice specific heat of a solid in a wide temperature range is usually described by Einstein model ${ }^{24}$. In this case, the position shift due to the temperature change can be predicted by Varshni equation of $q_{1}=q_{1}^{0}-\frac{S_{q}}{\exp \left(\theta_{q} / T\right)-1}$ (where $q_{1}^{0}$ is the first maximum position at $0 \mathrm{~K}, S_{q}$ and $\theta_{q}$ is a fitting parameter and the effective 
Einstein temperature, respectively. ${ }^{25}$. The fitting parameters are listed in Fig. 1d. The effective Einstein temperature of $349.95 \mathrm{~K}$ is consistent with the value $(369.89 \mathrm{~K})$ measured from the elastic modulus at different temperatures of the $\mathrm{BMG}^{26}$.

In order to further explore the structural evolution on the atomic scale, the structure information in real space is investigated. The calculation of the atomic pair correlation function (PDF) using the usual sine-Fourier transformation of the structure factors can plot $P D F(r)$ at different temperatures (Fig. 2a). The maxima in $P D F(r)$ correspond to different inter-atomic distances. Enlarged first maximum shows that the position shifts to larger $r$ values with decreasing temperature from $308 \mathrm{~K}$ to $98 \mathrm{~K}$ (inset of Fig. 2a). Using cubic polynomial function to fit the first maximum of the $P D F(r)$ curve (Fig. $\mathrm{S} 2$ ), the position can be obtained, and then plotted in Fig. 2b. It is evident that the first maximum position shifts from $3.081 \AA$ to 3.090 $\AA$ when the temperature is decreased. This suggests that the expansion of the first nearest neighbor shell upon cooling to low temperatures. Thus, we can reasonably conjecture that the radius of the first shell, $d_{0} / 2$, slightly expands approximately $0.3 \%$. For the other maxima, such as the second maximum, the reduction in the temperature causes that the position shifts to lower $r$ values (inset of Fig. 2a). The second maximum position measured from the cubic polynomial function fitting curve as a function of the temperature is plotted in Fig. 2c, which shows that the cooling shrinkage is approximately $0.2 \%$. The third and fourth maxima positions as functions of the temperature are shown in Fig. S2, which suggests that the positions of these two maxima also shift to lower $r$ values with decreasing temperature (Figs. S3b and d). It is obvious that except the first nearest neighbor shell, the other atomic shells shrink approximately $0.2 \%$ due to the temperature decreased from $308 \mathrm{~K}$ to $98 \mathrm{~K}$. These shrinkages of the maxima in high $r$ values in real space are consistent with the shrinkage of the first maximum in reciprocal space (Fig. 1d).

To reveal the structural information hidden in the first nearest neighbor shell of the BMG, the coordination numbers as functions of temperature will be investigated. The change of the first nearest neighbor shell with temperature is shown in Fig. 3a by comparing the first maxima of radial distribution functions, $R D F(r)$, at different temperatures. The first maximum in $R D F(r)$ is composed of two sub-peaks with maxima around 2.7 and $3.1 \AA$, respectively (Fig. 3a). From the type and concentration of the constituent elements in the $\mathrm{Zr}_{41.25} \mathrm{Ti}_{13.75} \mathrm{Ni}_{10} \mathrm{Cu}_{12.5} \mathrm{Be}_{22.5} \mathrm{BMG}$, the possible atomic pairs are listed in Table I. The weighting factors, $W_{i j}$, of the atomic pairs are calculated according to $W_{i j}=\frac{C_{i} f_{i} C_{j} f_{j}}{\left(\sum C_{i} f_{i}\right)^{2}}$, (where $C$ is the atomic number of element; $i$ and $j$ represent different elements; $f$ is the atomic fraction of element. $)^{27}$, which are listed in Table I. It is clear that $\mathrm{Zr}-\mathrm{Zr}, \mathrm{Zr}-\mathrm{Cu}, \mathrm{Zr}-\mathrm{Ti}$ and $\mathrm{Zr}-\mathrm{Ni}$ are the dominant atomic pairs that constitute the first coordination shell. For the other eleven pairs, the weighting factors are negligibly small due to low concentrations as well as their relatively weak scattering powers. The theoretical bond lengths are estimated from the covalent radii of the atoms (i.e. $R_{Z r}=1.60 \AA, R_{N i}=1.25 \AA, R_{B e}=1.12 \AA, R_{T i}=1.47 \AA$ and $R_{C u}=$ $1.28 \AA$ ), which are listed in Table I. Partial atomic pairs at different bond lengths are marked in Fig. 3a. It can be seen that the sub-peak I is mainly due to overlapping of $\mathrm{Zr}-\mathrm{Ni}$ and $\mathrm{Zr}$-Cu pairs, and the subpeak II is due to $\mathrm{Zr}-\mathrm{Zr}$ and $\mathrm{Zr}$-Ti pairs. Figure $3 \mathrm{a}$ clearly shows that besides the shift of the sub-peaks' positions, the intensities of the two sub-peaks also change with temperature. The areas of the two subpeaks of the first maximum in $R D F(r)$ are associated with the nearest neighbor atomic coordination numbers ${ }^{27}$. The averaged weighted
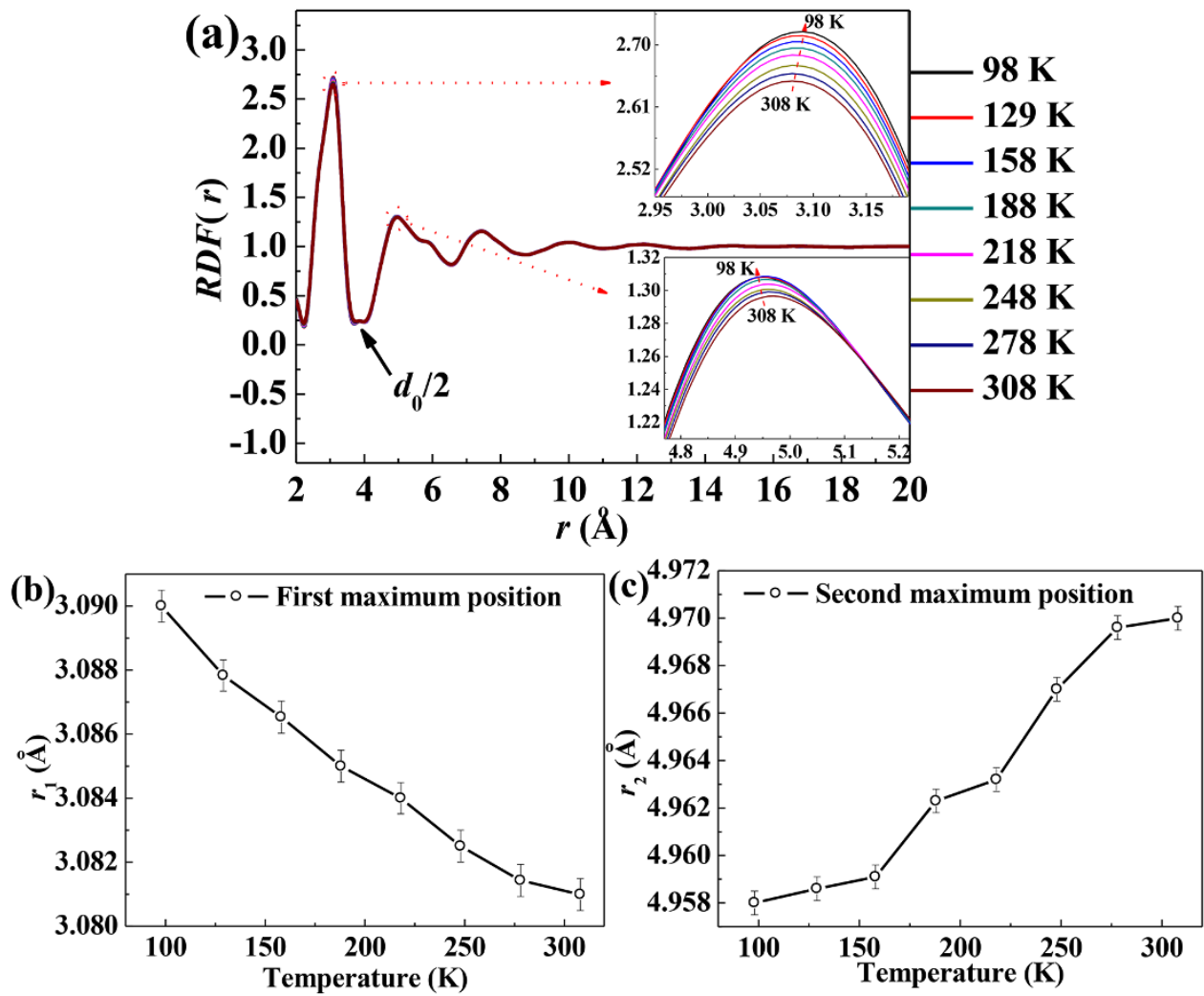

Figure $2 \mid$ Structural evolution in real space. (a) Pair correlation function, $\operatorname{PDF}(r)$, at different temperatures. (b) The position of first maximum of $\operatorname{PDF}(r)$ as a function of temperature. The position of first maximum in $P D F(r)$ is obtained by cubic polynomial function fitting curve, which is shown in Fig. S4. (c) The position of second maximum of $P D F(r)$ as a function of temperature. The position of first maximum in $P D F(r)$ is obtained by cubic polynomial function fitting curve. 

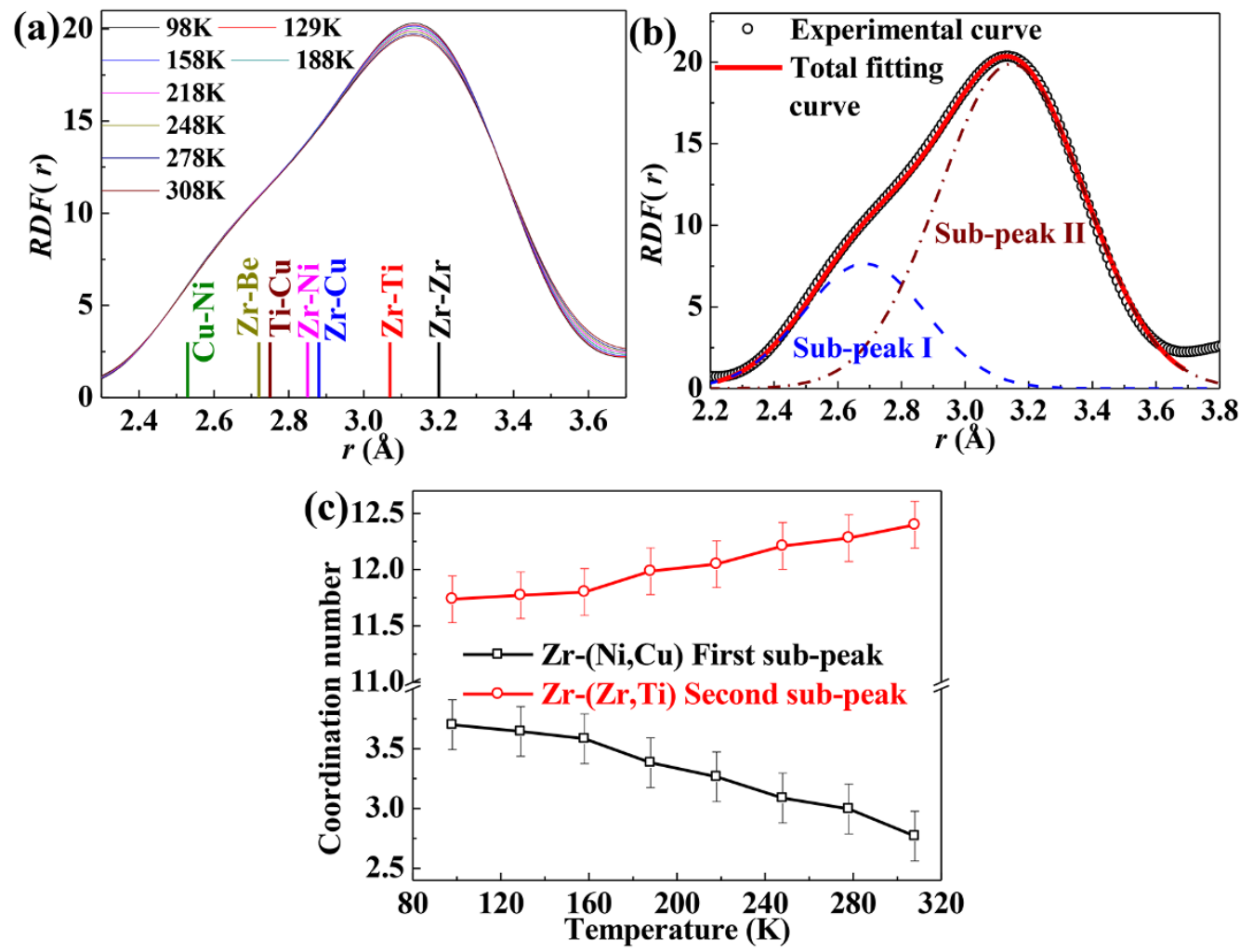

Figure 3 Coordination number in first nearest neighbor shell. (a) First maximum of radial distribution function, $R D F(r)$, at different temperatures. (b) Deconvolution of the first nearest neighbor shell into two Gaussians at $98 \mathrm{~K}$. (c) Coordination numbers versus temperature.

coordination numbers integrated by a fit of two Gaussian functions (Fig. 3b) are plotted in Fig. 3c as functions of temperature. Because of the systematic errors in the experiments and data evaluation, the accuracy of the coordination numbers is estimated as $\pm 10 \%{ }^{28}$. With increasing temperature, the coordination number of the $\mathrm{Zr}$ $(\mathrm{Zr}, \mathrm{Ti})$ atomic pairs increases and that of the $\mathrm{Zr}-(\mathrm{Ni}, \mathrm{Cu})$ atomic pairs decreases. The total coordination numbers in the first nearest neighbor shell, i.e., $\left(N_{\text {Sub-peakI }}+N_{\text {sub-peakII }}\right) / 2$, increase from $7.58 \pm 0.20$ to $7.72 \pm 0.20$ with decreasing temperature. However, this increment of the total coordination numbers is within the error range of \pm 0.20 . Thus, although it is not reasonable to accurately determine the coordination numbers, an increasing trend of the total coordination numbers is observed.

Table I | Fifteen possible atomic pairs in the $\mathrm{Zr}_{41.25} \mathrm{Ti}_{13.75} \mathrm{Ni}_{10^{-}}$ $\mathrm{Cu}_{12.5} \mathrm{Be}_{22.5} \mathrm{BMG}$, theoretical bond length of these atomic pairs and their weighting factors calculated at $q=0 \AA^{-1}$

\begin{tabular}{lcc} 
Atomic pair & Weighting factor & Bond length (Å) \\
\hline $\mathrm{Zr}-\mathrm{Zr}$ & 0.378 & 3.2 \\
$\mathrm{Zr}-\mathrm{Cu}$ & 0.166 & 2.88 \\
$\mathrm{Zr}-\mathrm{Ti}$ & 0.138 & 3.07 \\
$\mathrm{Zr}-\mathrm{Ni}$ & 0.128 & 2.85 \\
$\mathrm{Zr}-\mathrm{Be}$ & 0.041 & 2.72 \\
$\mathrm{Ti}-\mathrm{Cu}$ & 0.029 & 2.75 \\
$\mathrm{Cu}-\mathrm{Ni}$ & 0.028 & 2.53 \\
$\mathrm{Ti}-\mathrm{Ni}$ & 0.023 & 2.72 \\
$\mathrm{Cu}-\mathrm{Cu}$ & 0.018 & 2.56 \\
$\mathrm{Ti}-\mathrm{Ti}$ & 0.012 & 2.94 \\
$\mathrm{Ni}-\mathrm{Ni}$ & 0.011 & 2.50 \\
$\mathrm{Be}-\mathrm{Cu}$ & 0.009 & 2.40 \\
$\mathrm{Ti}-\mathrm{Be}$ & 0.007 & 2.59 \\
$\mathrm{Be}-\mathrm{Ni}$ & 0.007 & 2.37 \\
$\mathrm{Be}-\mathrm{Be}$ & 0.001 & 2.24 \\
\hline
\end{tabular}

\section{Discussion}

Previous research has revealed that elastic loading can trigger atomic rearrangements in the first nearest neighbor shell ${ }^{29}$. A bond reorientation model was deduced to describe the anisotropic structural evolution in $\mathrm{BMGs}^{28,29}$, suggesting that the atomic rearrangements in the first nearest neighbor shell initiate a concordant shift of the surrounding atoms. Along this line, a concordant shifting region is developed, and treated as the primary deformation units to deliver the plastic strain ${ }^{30-32}$. Our in-situ X-ray diffraction results upon cooling corroborate that expansion in the first nearest neighbor shell happens, which is attributed to the changes in the local structure of the polyhedra or in the atomic arrangement in the short-range order ${ }^{33}$. The coordination numbers slightly increase with decreasing temperature confirm that the structural changes in the first nearest neighbor shell (Fig. 3). These changes must influence the macroscopic mechanical behavior. Usually, the macroscopic behavior in materials is determined by the medium-range order ${ }^{34}$. Thus, the concordant shifting region concept can provide a bridge to correlate the cooling shrinkage in the medium-range order and the macroscopic mechanical properties.

According to the concordant region concept ${ }^{35}$, the local change (cooling shrinkage) in the short-range order must change the local energy concentration. To counterbalance this local energy change, surrounding atoms must shift cooperatively to distribute the energy changes $^{36}$, which can behave a liquid-like flow unit to carry the $\operatorname{strain}^{9,37}$. On the other hand, the formation of a concordant region can be treated as a process that the external stress is subjected to a redistribution of local stresses in an elastic medium ${ }^{38}$. This redistribution of local stresses is carried out by an elastic interaction between the atoms in a distance of shear wave propagation ${ }^{39}$. Thus, this distance can be calculated to be $d=V_{s} \tau$, where $V_{\mathrm{s}}$ is the shear wave speed, and $\tau$ is the relaxation time that depends on the temperature. In the present study, since the test temperature ranged from room temperature to approximately $100 \mathrm{~K}$, the $\tau$ value is a constant 
(approximately $1 \sim 2 \mathrm{ps})^{38}$, because the $\alpha$ relaxation of glassy phase cannot be influenced by the temperature well below the glass transition temperature ${ }^{38}$. The shear wave speed, $V_{s}$, depends on the mass density, $\rho$, and the shear modulus, $G$, i.e., $V_{\mathrm{s}}=(G / \rho)^{1 / 2}$. The cooling shrinkage causes the mass density to be larger, which can be deduced by the atomic volume, i.e. $\rho=M /\left(A_{\mathrm{v}} \cdot v_{\mathrm{a}}\right)$, where $A_{\mathrm{v}}$ is Avogadro constant and $M$ is the molar weight of the $\mathrm{Zr}_{41.25} \mathrm{Ti}_{13.75} \mathrm{Ni}_{10^{-}}$ $\mathrm{Cu}_{12.5} \mathrm{Be}_{22.5} \mathrm{BMG}(60.05 \mathrm{~g} / \mathrm{mol})$. The mass density of the BMG as a function of temperature is plotted in Fig. 4a. Meanwhile, the shear modulus of the BMG at different temperatures, $G(T)$, can be predicted by the Varshni equation ${ }^{25}$, i.e. $G(T)=G_{0}-\frac{S}{\exp (\theta / T)-1}$, (where $G_{0}$ is the shear modulus at $0 \mathrm{~K} ; S$ is an fitting parameter related to the strength of the anharmonic interactions and $\theta$ is the effective Einstein temperature. ${ }^{40}$. For the $\mathrm{Zr}_{41.25} \mathrm{Ti}_{13.75} \mathrm{Ni}_{10} \mathrm{Cu}_{12.5^{-}}$ $\mathrm{Be}_{22.5} \mathrm{BMG}$, our previous study has found that the $G_{0}, S$ and $\theta$ values are $35.43 \mathrm{GPa}, 8.40$ and $365.89 \mathrm{~K}$, respectively ${ }^{26}$. From these correlations, the $d$ value as a function of temperature can be deduced (Fig. 4a). It is obvious that the size (volume) of the concordant shifting region increases with decreasing temperature. Thus, the number of atoms participating in concordant shift increases. When the BMG is driven by stress, the concordantly shifting atoms will store the elastic energy until yielding occurs. The number of atoms in the volume of the concordantly shifting region, i.e., the volume of the associated group of atoms, is an important factor influencing the macroscopic mechanical properties. Thus, it is required to further characterize the stress increment, $\Delta p$, resulting from concordant shifting, which can be expressed $\mathrm{as}^{31}$, $\Delta p=\frac{\pi}{2} \Delta p_{0} d_{0}{ }^{3} \rho \ln \frac{2 d}{d_{0}}$ (where the $\Delta p_{0}$ value is a constant, which reflects the stress increment in the first shell; $d_{0}$ is the diameter of the first shell; $\rho$ is mass density; $d$ is the concordant region size. $)^{38}$. Although, direct measurement of the $d_{0}$ value is not possible, we can use the position of the first maximum in the PDF curve to characterize the relative changes of the $d_{0}$ values at different temperature (Fig. 2a)
Since the $\Delta p_{0}$ value cannot be calculated, we used the ratio of $\Delta p /$ $\Delta p_{0}$ to reflect the stress increment in the concordantly shifting region. The $\Delta p / \Delta p_{0}$ value as a function of the $d$ value is plotted in Fig. 4b. The ratio monotonically increases from negative to positive values with increasing $d$. It can be concluded that the change from negative to positive values occurs at the radius of the first nearest neighbor shell of the BMG (Fig. 4b). According to the concept of concordant local rearrangement events ${ }^{29}$, the atomic bond shrinkage of the atoms in the first nearest neighbor shell approaches a potential minimum upon cooling, causing that the first nearest neighbor shell supports less stress than at room temperature. As such, the stress increment, $\Delta p$, exhibits a negative value. Beyond the first shell (higher than $\sim 3.7 \AA$ ), a positive ratio, i.e., a positive stress increment, appears because the surrounding atomic layers must counterbalance the reduction of the local stress in the first nearest-neighbor shell and then support more stress. At the boundary of the concordant region (which is determined by the shear wave speed), the $\Delta p / \Delta p_{0}$ ratios approach a maximum value (see the inset in Fig. $4 \mathrm{~b}$ ). This is closely associated with the activation energy for atomic rearrangements in the concordant region ${ }^{35}$. The increase of the activation energy of the concordant region is affected by the volume, $v$, and the stress increment, i.e., $\Delta e=v \Delta p$, where $v=(4 \pi / 3) d^{335}$. The temperature decreasing from $308 \mathrm{~K}$ to $98 \mathrm{~K}$ causes that the activation energy of the concordantly shifting region increases by approximately $19 \%$. The compression tests of the BMG in the temperature range from room temperature down to $123 \mathrm{~K}$ show that the yield strength of the BMG increases by about $17 \%$, which is plotted in Fig. 4c. For comparison, the yield strength as a function of temperature deduced by the activation energy of the concordant region is also potted in Fig. 4c. It can be seen that the experimental values almost follow the values deduced from the concordant region model. Therefore, we assume that the concordant region operation is a primary mechanism influencing the macro-mechanical behavior. The expansion of the concordant region causes its activation energy increment to increase, which strengthens glassy materials. In this case, when the temperature is kept at a low temperature, such as $98 \mathrm{~K}$, external stress is applied to
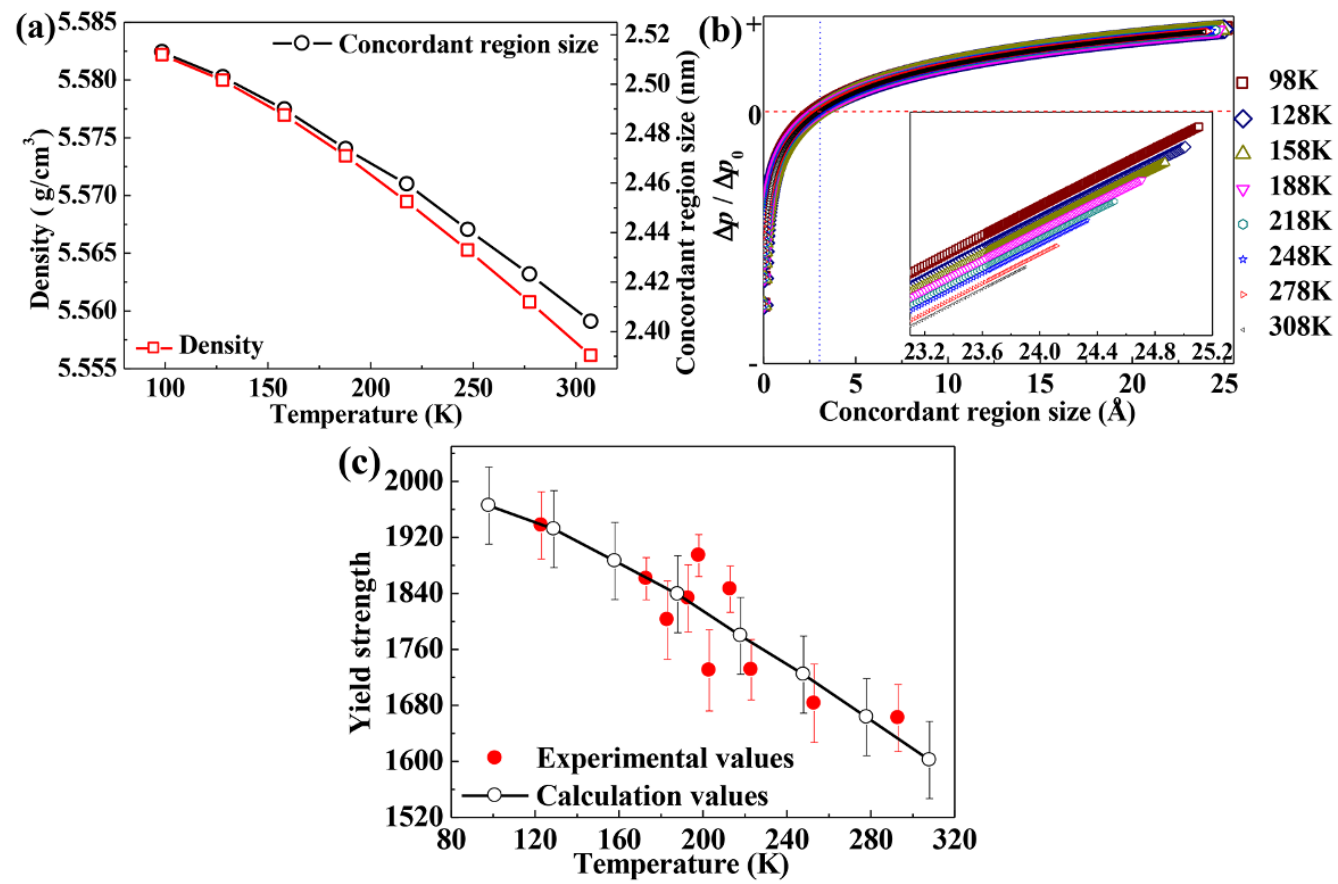

Figure $4 \mid$ Relationship between atomic structural evolution and yield strength. (a) Mass density and concordant region size, $d$, of the BMG as functions of temperature. (b) Relationship between the ratio of $\Delta p / \Delta p_{0}$ and the $d$ value of the BMG at different temperatures. The inset shows an enlarged view. (c) The yield strengths deduced by the activation energy of concordant region and measured by compression tests, respectively. 
the BMG cannot change the activation energy increment ${ }^{31}$. Thus, the external stress does not influence the strength of the BMG.

Previous in-situ ultrasonic measurements have found that the Varshni equation could well describe the elastic constants of BMGs at different temperatures ${ }^{40}$. From this equation, the elastic moduli monotonically increase with decreasing temperature, suggesting that BMGs become more stiffer at lower temperatures. In this case, cohesive strength between atoms of BMGs increases due to cooling ${ }^{40}$, which causes the yield strength of BMGs increasing at lower temperatures. This further confirms that the current concept of the concordantly shifting region.

In summary, the structural evolution on the atomic scale of the $\mathrm{Zr}_{41.25} \mathrm{Ti}_{13.75} \mathrm{Ni}_{10} \mathrm{Cu}_{12.5} \mathrm{Be}_{22.5}$ BMG with decreasing temperature down to $98 \mathrm{~K}$ was investigated by in-situ high energy X-ray synchrotron radiation and compression tests at low temperature. A concept of a concordant region was introduced to elucidate the correlation between the atomic behavior and the fracture strength of the BMG, and the activation energy increment at lower temperature is considered as the main factor influencing the fracture strength. A larger concordant region size and a larger stress increment contribute to a higher strength.

\section{Methods}

Alloy ingots with nominal compositions of $\mathrm{Zr}_{41.25} \mathrm{Ti}_{13.75} \mathrm{Ni}_{10} \mathrm{Cu}_{12.5} \mathrm{Be}_{22.5}$ were prepared by arc melting appropriate mixtures of pure metals in a Ti-gettered argon atmosphere, followed by suction casting into a copper mold to form BMG rod-like samples with a size of $\Phi 1 \times 20 \mathrm{~mm}$. Samples with a geometry size of $\Phi 1 \times 2 \mathrm{~mm}$, i.e., with an aspect ratio of $2: 1$, were cut from the BMG rods by a diamond saw, and the ends of the specimens were carefully polished to be parallel.

In-situ XRD experiments were performed at the P02.1 beamline of the PETRA III electron storage ring (Hamburg, Germany). The samples were placed inside a quartz capillary (1.2 $\mathrm{mm}$ in diameter, $20 \mu \mathrm{m}$ in wall thickness). The sample was cooled by liquid nitrogen. The sample sealed in quartz capillary was fixed by a sample holder that was not influenced by liquid nitrogen. The diffraction center, which was located in the center of the rod-like sample, was in a fixed position because the sample shrank homogeneously. The sample to detector distance and the orthogonality of the detector with respect to the incident beam were calibrated using the reference material LaB6 (NIST 660a). The sample to detector distance was $358.099 \mathrm{~mm}$. The beam size was $0.6 \times 0.6 \mu \mathrm{m}^{2}$ and the wavelength was $0.20727 \AA$. The diffracted photons were collected with a 2D detector (Perkin Elmer PE1621). Cooling from room temperature down to approximately $98 \mathrm{~K}$ was carried out at a cooling rate of $5 \mathrm{~K} / \mathrm{min}$. Two-dimensional X-ray diffraction patterns were radically integrated using the program FIT2 ${ }^{41}$.

1. Ritchie, R. O. The conflicts between strength and toughness. Nature Mater. 10 817-822 (2011).

2. Tan, J. et al. Correlation between internal states and plasticity in bulk metallic glass. Appl. Phys. Lett. 98, 151906 (2011)

3. Wang, G., Mattern, N., Pauly, S., Bednarčík, J. \& Eckert, J. Atomic structure evolution in bulk metallic glass under compressive stress. Appl. Phys. Lett. 95, 251906 (2009)

4. Chen, M. W. Mechanical behavior of metallic glasses: Microscopic understanding of strength and ductility. Annu. Rev. Mater. Res. 38, 445-469 (2008).

5. Miracle, D. B. A structural model for metallic glasses. Nature Mater. 3, 697-702 (2004)

6. Yavari, A. R., Lewandowski, J. J. \& Eckert, J. Mechanical properties of bulk metallic glasses. MRS Bull. 32, 635-638 (2007)

7. Spaepen, F. A microscopic mechanism for steady state inhomogeneous flow in metallic glasses. Acta Metall. 25, 407-415 (1977).

8. Argon, A. S. Plastic deformation in metallic glasses. Acta Metall. 27, 47-58 (1979)

9. Ye, J. C., Lu, J., Liu, C. T., Wang, Q. \& Yang, Y. Atomistic free-volume zones and inelastic deformation of metallic glasses. Nature Mater. 9, 619-623 (2010).

10. Pan, D., Inoue, A., Sakurai, T. \& Chen, M. W. Experimental characterization of shear transformation zones for plastic flow of bulk metallic glasses. Proc. Natl Acad. Sci. USA 105, 14769-14772 (2008).

11. Sheng, H. W., Luo, W. K., Alamgir, F. M., Bai, J. M. \& Ma, E. Atomic packing and short-to-medium-range order in metallic glasses. Nature 439, 419-425 (2006)

12. Wang, Z., Wen, P., Huo, L. S., Bai, H. Y. \& Wang, W. H. Signature of viscous flow units in apparent elastic regime of metallic glasses. Appl. Phys. Lett. 101, 121906-121904 (2012).

13. Pan, D. et al. Correlation between structural relaxation and shear transformation zone volume of a bulk metallic glass. Appl. Phys. Lett. 95, 141909 (2009).

14. Li, H., Tao, K., Fan, C., Liaw, P. K. \& Choo, H. Effect of temperature on mechanical behavior of Zr-based bulk metallic glasses. Appl. Phys. Lett. 89, 041921041921-041923 (2006)
15. Fan, C. et al. Mechanical behavior of a Zr-based bulk metallic glass and its composite at cryogenic temperatures. J. Mater. Res. 22, 445-452 (2007).

16. Fan, C. et al. Mechanical behavior of bulk amorphous alloys reinforced by ductile particles at cryogenic temperatures. Phys. Rev. Lett. 96, 145506 (2006).

17. Li, H., Fan, C., Tao, K., Choo, H. \& Liaw, P. K. Compressive Behavior of a Zr-Based Metallic Glass at Cryogenic Temperatures. Adv. Mater. 18, 752-754 (2006).

18. Li, C. J. et al. Enhanced strength and transformation-induced plasticity in rapidly solidified Zr-Co-(Al) alloys. Scr. Mater. 68, 897-900 (2013).

19. Li, C. J. et al. On the transformation-induced work-hardening behavior of $\mathrm{Zr}_{47.5} \mathrm{Co}_{47.5} \mathrm{Al}_{5}$ ultrafine-grained alloy. Intermetallics 35, 116-119 (2013).

20. Stoica, M. et al. Strain distribution in $\mathrm{Zr}_{64.13} \mathrm{Cu}_{15.75} \mathrm{Ni}_{10.12} \mathrm{Al}_{10}$ bulk metallic glass investigated by in situ tensile tests under synchrotron radiation. J. Appl. Phys. 104, 013522 (2008).

21. Bednarcik, J., Michalik, S., Kolesar, V., Rütta, U. \& Franz, H. In situ XRD studies of nanocrystallization of Fe-based metallic glass: a comparative study by reciprocal and direct space methods. Phys. Chem. Chem. Phys. 15, 8470-8479 (2013).

22. Ma, D., Stoica, A. D. \& Wang, X. L. Power-law scaling and fractal nature of medium-range order in metallic glasses. Nature Mater. 8, 30-34 (2009).

23. Grüneisen, E. Theorie des festen Zustandes einatomiger Elemente. Annalen der Physik 39, 257-306 (1912).

24. Fabian, J. \& Allen, P. B. Thermal expansion and Grüneisen parameters of amorphous silicon: A realistic model calculation. Phys. Rev. Lett. 79, 1885 (1997)

25. Varshni, Y. P. Temperature dependence of the elastic constants. Phys. Rev. B 2, 3952 (1970).

26. Liu, Z. Y. et al. Low temperature dependent dynamics transition of intermittent plastic flow in a metallic glass. I. Experimental investigations. J. Appl. Phys. 114, 033520 (2013).

27. Egami, T. \& Billinge, S. J. L. Underneath the Bragg Peaks: Structural Analysis of Complex Materials (Pergamon, New York/Elsevier, Oxford, England) 2003

28. Mattern, N. et al. Structural evolution of $\mathrm{Cu}-\mathrm{Zr}$ metallic glasses under tension. Acta Mater. 57, 4133-4139 (2009).

29. Suzuki, Y., Haimovich, J. \& Egami, T. Bond-orientational anisotropy in metallic glasses observed by x-ray diffraction. Phys. Rev. B 35, 2162-2168 (1987).

30. Wang, G. et al. Deformation induced structural evolution in bulk metallic glasses. Chin. Sci. Bull. 56, 3952-3959 (2011).

31. Wang, G. et al. Correlation between elastic structural behavior and yield strength of metallic glasses. Acta Mater. 60, 3074-3083 (2012).

32. Liu, Z. Y. et al. Low temperature dependent dynamics transition of intermittent plastic flow in a metallic glass. II. Dynamics analysis. J. Appl. Phys. 114, 033521 (2013).

33. Lou, H. et al. Negative expansions of interatomic distances in metallic melts. Proc. Natl. Acad. Sci. U. S. A. 110, 10068-10072 (2013).

34. Schuh, C. A., Hufnagel, T. C. \& Ramamurty, U. Mechanical behavior of amorphous alloys. Acta Mater. 55, 4067-4109 (2007).

35. Trachenko, K. The Vogel-Fulcher-Tammann law in the elastic theory of glass transition. J. Non-Cryst. Solids 354, 3903-3906 (2008).

36. Goldstein, M. Viscous Liquids and the Glass Transition: A Potential Energy Barrier Picture. J. Chem. Phys. 51, 3728-3739 (1969).

37. Wang, Z., Wen, P., Huo, L. S., Bai, H. Y. \& Wang, W. H. Appl. Phys. Lett. 101, 121906 (2012).

38. Trachenko, K. Slow dynamics and stress relaxation in a liquid as an elastic medium. Phys. Rev. B 75, 212201 (2007).

39. Levashov, V. A., Morris, J. R. \& Egami, T. Viscosity, shear waves, and atomic-level stress-stress correlations. Phys. Rev. Lett. 106, 115703 (2011).

40. Yu, P., Wang, R. J., Zhao, D. Q. \& Bai, H. Y. Temperature dependence of elastic moduli in bulk metallic glasses down to liquid nitrogen temperature. Appl. Phys. Lett. 90, 251904 (2007).

41. Hammersley, A. P., Svensson, S. O., Hanfland, M., Fitch, A. N. \& Häusermann, D. High Press. Res. 14, 235 (1996).

\section{Acknowledgments}

The stimulated discussions with Professor W.H. Wang at the Institute of Physics, CAS, are acknowledged. The work was supported by the Alexander von Humboldt Foundation and the NSF of China (Nos. 51171098, 51222102, and 51301078). G. Wang also thanks the financial support by the Innovation Program of Shanghai Municipal Education Commission (No. 12ZZ090), and the Program for Professor of Special Appointment (Eastern Scholar) at Shanghai Institutions of Higher Learning and the 085 project in Shanghai University.

\section{Author contributions}

J.T. and G.W. conceived and designed the experiments. J.T., G.W., Z.Y.L., J.B., Y.L.G., Q.J.Z performed the experiments. J.T., G.W. and J.B. analyzed the data. J.T., G.W., N.M. and J.E. wrote the main manuscript text. J.T. and G.W. prepared the figures and tables. All authors reviewed the manuscript.

\section{Additional information}

Supplementary information accompanies this paper at http://www.nature.com/ scientificreports 
Competing financial interests: The authors declare no competing financial interests.

How to cite this article: Tan, J. et al. Correlation between atomic structure evolution and strength in a bulk metallic glass at cryogenic temperature. Sci. Rep. 4, 3897; DOI:10.1038/ srep03897 (2014). (c) (i) (2) This work is licensed under a Creative Commons AttributionBy NC SA NonCommercial-ShareAlike 3.0 Unported license. To view a copy of this license, visit http://creativecommons.org/licenses/by-nc-sa/3.0 